\title{
Comparative Study on the Axial Compression and Bearing Capacity of Reactive Powder Concrete-Filled Circular Steel Tube
}

\author{
Mingyang Chen (D) and Xiaomeng Hou \\ Department of Civil Engineering, Harbin Institute of Technology, Harbin, China \\ Correspondence should be addressed to Mingyang Chen; chenmy08@163.com
}

Received 29 May 2018; Accepted 4 December 2018; Published 19 December 2018

Guest Editor: Munir Nazzal

Copyright (c) 2018 Mingyang Chen and Xiaomeng Hou. This is an open access article distributed under the Creative Commons Attribution License, which permits unrestricted use, distribution, and reproduction in any medium, provided the original work is properly cited.

\begin{abstract}
Reactive powder concrete (RPC) was confined by the circular steel tube to obtain the required ductility. The axial compression test results of 139 columns from different scholars were collated and compared to study the axial compression and bearing capacity of a reactive powder concrete-filled circular steel tube, of which the confining coefficient is $0.057-2.312$ and the RPC strength is 76.6-178.2 MPa. Load-displacement curves have been categorized into four stages: (1) elastic; (2) elastic-plastic; (3) descending; and (4) strengthening. The failure mode can be divided into three types according to the different confining coefficients as (1) wall buckling; (2) diagonal shear; and (3) drum-shaped. The confining coefficient, core RPC strength, steel fiber volume, steel tube $D / t$ ratio, and loading mode on the ultimate bearing capacity were analyzed. The results showed the confining coefficient to be the main factor affecting ultimate bearing capacity. The equation for determining ultimate bearing capacity was established based on the limit equilibrium theory, with the lateral confining coefficient of RPC $(k)$ determined to be 2.86, less than that of normal concrete at 4.1. Based on the experimental analysis results and China's "Design and Construction Code for Concrete-Filled Steel Tube Structure" (CECS 28-2012), the design proposal for an RPC-filled steel tube was recommended.
\end{abstract}

\section{Overview of Experiments}

Reactive powder concrete (RPC) is the direction for further development of high-strength concrete (HSC) and ultrahigh performance concrete (UHPC), with a relatively new type of cement-based composite material successfully developed by Richard in 1993, which has ultrahigh strength, good volume stability, and excellent durability $[1,2]$. The science behind the development of RPC is mature, and many studies have focused on its mechanical properties for further broad application in engineering [3-5]. However, RPC is very dense and uniform since it does not contain coarse aggregate; therefore, the pinning effect provided by coarse aggregates in normal concrete (NC) is weakened or even absent in RPC, and most RPCs are "explosive" and are destroyed by topdown longitudinal splitting after compression [5]. Measures to decrease brittleness and increase ductility of RPC are used for structures with ductile seismic performance requirements. RPC has been used in concrete-filled steel tubes
(RPCFTs) [6]. The steel tube restrains transverse deformation of the core RPC to allow the superior compressive performance of RPC to be fully utilized and its ductility to meet the requirements of the column design. The successful application of steel tube RPC in the construction of the Sherbrooke Bridge, Quebec, Canada, demonstrated its superior mechanical properties and economic benefits [7]. Although both RPC and NC are cement-based composite materials, their water/binder ratios, composition of raw materials, and mechanical properties differ considerably [8]. Therefore, the theory and method for calculating the ultimate bearing capacity of CFT cannot be directly applied to RPCFT.

The present study aimed at analyzing the axial compressive mechanical behavior and ultimate bearing capacity of RPCFTs. Many studies have focused on the experimental determination of the axial compression performance and ultimate bearing capacity of RPCFTs. The present study collated the experimental results of past studies on the axial 
compression performance of 139 RPCFTs during 2003 to 2016, including Meng Shiqiang [8], Zhang Jing [9], Yang Wusheng [10], Wu Yanhai [11], Feng Jianwen [12], Yan Zhigang [13], Luo Hua [14], Yang Guojing [15], and Jian Wei [16]. Among the studies, tube diameter, wall thickness, and strength varying between $102 \mathrm{~mm}$ and $273 \mathrm{~mm}, 1 \mathrm{~mm}$ and $12 \mathrm{~mm}$, and $210 \mathrm{MPa}$ and $425 \mathrm{MPa}$, respectively, were considered to cause variation of the steel tube confining coefficient $(\xi)$ in the range of $0.057-2.312$. RPC strengths in the range of $76.6 \mathrm{MPa}-178.2 \mathrm{MPa}$ were affected by the RPC water/binder ratio (0.16-0.26), RPC steel fiber content (0\%-2\%), and curing methods (standard curing, $90^{\circ} \mathrm{C}$ steam curing, and curing in an autoclaved reactor with a maximum temperature and pressure of $200^{\circ} \mathrm{C}$ and $1.3 \mathrm{MPa}$, respectively). The main parameters are shown in Table 1.

\section{Test Results}

2.1. Load-Displacement Relationship. A review of the 139 RPCFT axially compressed experiments conducted in past studies found that the vertical stress and strain of the steel tube and core RPC gradually increased with increasing axial load due to pressure, and the core RPC was compressed in three directions because of constraints placed by the steel tube. The RPC peak strain under pressure varied from 0.0035 to 0.008 and increased by $3 \%$ to $32 \%$, respectively. The ultimate bearing capacity of RPCFT increased between $2 \%$ and $58 \%$ over the sum of the bearing capacity of the steel tube and RPC, and brittle failure of the core RPC was not found with effective restraint, plastic deformability of the components was gradually reduced, and the integrity of samples with larger confining coefficients remained after sizeable deformations of $5 \%$, thereby demonstrating excellent ductility. Figure 1 shows the load-displacement curve of the specimen. The mechanical characteristics of RPCFT could be divided into four stages: (1) elasticity; (2) elasticplastic; (3) descending; and (4) strengthening.

2.1.1. Elastic Stage (OA). Vertical deformation of the steel tube and RPC increased proportionally with increased load, and no significant deformation of the steel tube surface was observed. The proportional ultimate load of RPCFT ranged from $85 \%$ to $95 \%$ of the ultimate bearing capacity, whereas the value of CFT was 70\%, smaller than that of RPCFT, thereby explaining the disproportionately high strength of RPC in relation to NC. RPC had a uniform and denser internal structure, with mechanical properties closer to elastic material. RPC remained elastic at the ultimate load of $80-95 \%$ with no significant volume expansion and maintained Poisson's ratio at $0.2-0.4$. CFT at $40 \%$ of the ultimate load experienced rapid expansion of the cracks in the interface between aggregate and cementite, with sharp increases in deformation and significant expansion of volume, resulting in the appearance of the Lüder $\mathrm{J}$ slip line. Figure 2 compares the longitudinal and transverse strains between RPC and NC.

2.1.2. Elastic-Plastic Stage $(A B)$. With the increasing axial load, yielding of the steel tube occurred due to compression and redistribution of internal force between RPC and steel tube. Thus, there were sharp increases in RPC compressive stress and internal microcracks emerged. In addition, there was continuous expansion of the widths and lengths of cracks, along with increases in transverse volume expansion deformation. The overall stiffness was degraded and the load-deformation curve showed a significant nonlinearity. Since RPC does not contain coarse aggregates, the fracture surface was relatively smooth, the steel fiber at the fracture surface was almost broken, and cracks were filled with powdery material. After the steel tube was extruded, the surface was partially convex, the rust began to peel off, and the Lüder J slip line appeared.

2.1.3. Descending Stage (BC). At the attainment of the ultimate load, a large amount of internal energy accumulated inside the core of RPC due to compression deformation being suddenly released. Internal cracks rapidly expanded to form a fracture surface. When the steel fiber was almost broken at the fracture surface, the strain energy was rapidly released and the sample broke into pieces. A sound of a sudden splitting failure could then be heard, indicating a load-deformation curve on the decline in the bearing capacity (BC). The sudden drop of bearing capacity involved a process where strain energy was rapidly released, which was dependent on the confining coefficient. RPCFT with a small confining coefficient experienced a small confining force provided by the steel tube. After reaching the ultimate load, the drop in longitudinal stress in the steel tube was higher than that of the restraining effect on the RPC strength provided by the steel tube. There was a marked drop in bearing capacity, and the slope ratio of the descending segment became almost equal to the RPC. An increasing confining coefficient resulted in a significant increase in the confinement effect of the steel tube on the core RPC, and the load-deformation curve showed that the load amplitude did not descend obviously or no obvious decrease after reaching the ultimate bearing capacity was evident. Taking specimens XG1-1 and G1 of Wu Yanhai [11] as examples, the confining coefficient of XG1-1 was small; thus, the steel tube had little restraining effect on RPC and the specimen emitted a bursting sound after reaching the ultimate load. The bearing capacity then drastically dropped and the remaining bearing capacity became only $65 \%$ of the ultimate load. The specimen showed significant brittle failure, whereas G1 had a large confining coefficient, the steel tube had a strong constraint on the RPC and the ultimate load bearing capacity descended slowly. The remaining bearing capacity reached as high as $95 \%$ of the ultimate load, the curve of the descending section was gentle, and the specimen underwent ductile failure.

2.1.4. Strengthening Stage (CD). The steel tube entered the strengthening stage after the plastic flow stage under a sufficiently thick steel tube wall, and the strong restraint provided by the steel tube continued to slow down the decline in the core RPC bearing capacity. The entire test bearing capacity entered a rebound state when the increased 
TABLE 1: The main parameters of the axial compression experiments of reactive powder concrete-filled steel tubes (RPCFTs)

\begin{tabular}{|c|c|c|c|c|c|c|}
\hline Index & Section & $f_{\mathrm{cu}}(\mathrm{MPa})$ & $f_{\mathrm{y}}(\mathrm{MPa})$ & $\xi$ & Fiber $(\%)$ & $N_{\mathrm{u}}(\mathrm{kN})$ \\
\hline 1 & $100 \times 4.5 \times 200$ & 141.5 & 210 & 0.434 & 2.00 & 2178 \\
\hline 2 & $100 \times 4.5 \times 200$ & 141.5 & 210 & 0.434 & 0.00 & 2070 \\
\hline 3 & $100 \times 4.5 \times 200$ & 141.5 & 210 & 0.434 & 2.00 & 2121 \\
\hline 4 & $125 \times 1 \times 438$ & 155.4 & 250 & 0.057 & 0.00 & 1700 \\
\hline 5 & $125 \times 1 \times 438$ & 155.4 & 250 & 0.057 & 0.00 & 2000 \\
\hline 6 & $125 \times 1 \times 438$ & 155.4 & 250 & 0.057 & 0.00 & 1840 \\
\hline 7 & $127 \times 2 \times 445$ & 155.4 & 245 & 0.113 & 0.00 & 1930 \\
\hline 8 & $127 \times 2 \times 445$ & 155.4 & 245 & 0.113 & 0.00 & 1790 \\
\hline 9 & $127 \times 2 \times 445$ & 155.4 & 245 & 0.113 & 0.00 & 1850 \\
\hline 10 & $133 \times 3.5 \times 465$ & 155.4 & 289 & 0.231 & 0.00 & 2420 \\
\hline 11 & $133 \times 3.5 \times 465$ & 155.4 & 289 & 0.231 & 0.00 & 2360 \\
\hline 12 & $133 \times 3.5 \times 465$ & 155.4 & 289 & 0.231 & 0.00 & 2490 \\
\hline 13 & $133 \times 5 \times 465$ & 155.4 & 319 & 0.378 & 0.00 & 2450 \\
\hline 14 & $133 \times 5 \times 465$ & 155.4 & 319 & 0.378 & 0.00 & 2480 \\
\hline 15 & $133 \times 5 \times 465$ & 155.4 & 319 & 0.378 & 0.00 & 2350 \\
\hline 16 & $133 \times 3.5 \times 465$ & 167.1 & 289 & 0.221 & 0.00 & 2180 \\
\hline 17 & $133 \times 3.5 \times 465$ & 167.1 & 289 & 0.221 & 0.00 & 2480 \\
\hline 18 & $133 \times 3.5 \times 465$ & 167.1 & 289 & 0.221 & 0.00 & 2440 \\
\hline 19 & $133 \times 3.5 \times 400$ & 167.1 & 289 & 0.221 & 0.00 & 2430 \\
\hline 20 & $133 \times 3.5 \times 400$ & 167.1 & 289 & 0.221 & 0.00 & 2500 \\
\hline 21 & $133 \times 3.5 \times 400$ & 167.1 & 289 & 0.221 & 0.00 & 2565 \\
\hline 22 & $102 \times 1 \times 324$ & 148 & 328 & 0.101 & 0.00 & 1310 \\
\hline 23 & $102 \times 1 \times 324$ & 172 & 328 & 0.088 & 1.20 & 1335 \\
\hline 24 & $104 \times 2 \times 416$ & 172 & 328 & 0.178 & 1.20 & 1463 \\
\hline 25 & $104 \times 2 \times 416$ & 172 & 328 & 0.178 & 1.20 & 1486 \\
\hline 26 & $106 \times 3 \times 378$ & 172 & 328 & 0.27 & 1.20 & 1780 \\
\hline 27 & $106 \times 3 \times 378$ & 172 & 328 & 0.27 & 1.20 & 1853 \\
\hline 28 & $106 \times 3 \times 378$ & 172 & 328 & 0.27 & 1.20 & 1760 \\
\hline 29 & $121 \times 5 \times 484$ & 172 & 350 & 0.439 & 1.20 & 2608 \\
\hline 30 & $121 \times 5 \times 484$ & 172 & 350 & 0.439 & 1.20 & 2576 \\
\hline 31 & $121 \times 5 \times 484$ & 172 & 350 & 0.439 & 1.20 & 2612 \\
\hline 32 & $128 \times 2 \times 466$ & 172 & 315 & 0.138 & 1.20 & 2256 \\
\hline 33 & $128 \times 2 \times 466$ & 172 & 315 & 0.138 & 1.20 & 2228 \\
\hline 34 & $128 \times 2 \times 466$ & 172 & 315 & 0.138 & 1.20 & 2218 \\
\hline 35 & $128 \times 2 \times 466$ & 172 & 315 & 0.138 & 1.20 & 2224 \\
\hline 36 & $128 \times 2 \times 466$ & 148 & 315 & 0.158 & 0.00 & 2190 \\
\hline 37 & $128 \times 2 \times 466$ & 148 & 315 & 0.158 & 0.00 & 2213 \\
\hline 38 & $130 \times 3 \times 455$ & 172 & 315 & 0.208 & 1.20 & 2496 \\
\hline 39 & $130 \times 3 \times 455$ & 172 & 315 & 0.208 & 1.20 & 2510 \\
\hline 40 & $130 \times 3 \times 455$ & 172 & 315 & 0.208 & 1.20 & 2523 \\
\hline 41 & $130 \times 3 \times 455$ & 172 & 315 & 0.208 & 1.20 & 2508 \\
\hline 42 & $130 \times 3 \times 455$ & 172 & 315 & 0.208 & 1.20 & 2489 \\
\hline 43 & $130 \times 3 \times 455$ & 172 & 315 & 0.208 & 1.20 & 2467 \\
\hline 44 & $133 \times 3 \times 400$ & 122.4 & 290 & 0.257 & 0.00 & 2000 \\
\hline 45 & $133 \times 3 \times 400$ & 122.4 & 290 & 0.257 & 0.00 & 2005 \\
\hline 46 & $133 \times 3 \times 400$ & 172.2 & 290 & 0.182 & 0.00 & 2300 \\
\hline 47 & $133 \times 3 \times 400$ & 172.2 & 290 & 0.182 & 0.00 & 2350 \\
\hline 48 & $133 \times 4.5 \times 400$ & 122.4 & 318 & 0.439 & 0.00 & 2250 \\
\hline 49 & $133 \times 4.5 \times 400$ & 122.4 & 318 & 0.439 & 0.00 & 2200 \\
\hline 50 & $133 \times 4.5 \times 400$ & 172.2 & 318 & 0.311 & 0.00 & 2700 \\
\hline 51 & $133 \times 4.5 \times 400$ & 172.2 & 318 & 0.311 & 0.00 & 2750 \\
\hline 52 & $133 \times 6.5 \times 400$ & 122.4 & 318 & 0.666 & 0.00 & 2300 \\
\hline 53 & $133 \times 6.5 \times 400$ & 122.4 & 318 & 0.666 & 0.00 & 2350 \\
\hline 54 & $133 \times 6.5 \times 400$ & 172.2 & 318 & 0.472 & 0.00 & 2950 \\
\hline 55 & $133 \times 6.5 \times 400$ & 172.2 & 318 & 0.472 & 0.00 & 2950 \\
\hline 56 & $133 \times 8.5 \times 400$ & 122.4 & 290 & 0.837 & 0.00 & 2500 \\
\hline 57 & $133 \times 8.5 \times 400$ & 122.4 & 290 & 0.837 & 0.00 & 2550 \\
\hline 58 & $133 \times 8.5 \times 400$ & 172.2 & 290 & 0.592 & 0.00 & 2950 \\
\hline 59 & $133 \times 8.5 \times 400$ & 172.2 & 290 & 0.592 & 0.00 & 2960 \\
\hline 60 & $133 \times 10 \times 400$ & 122.4 & 376 & 1.329 & 0.00 & 3200 \\
\hline
\end{tabular}


TABle 1: Continued.

\begin{tabular}{|c|c|c|c|c|c|c|}
\hline Index & Section & $f_{\text {cu }}(\mathrm{MPa})$ & $f_{\mathrm{y}}(\mathrm{MPa})$ & $\xi$ & Fiber $(\%)$ & $N_{\mathrm{u}}(\mathrm{kN})$ \\
\hline 61 & $133 \times 10 \times 400$ & 122.4 & 376 & 1.329 & 0.00 & 3100 \\
\hline 62 & $133 \times 10 \times 400$ & 172.2 & 376 & 0.941 & 0.00 & 3450 \\
\hline 63 & $133 \times 10 \times 400$ & 172.2 & 376 & 0.941 & 0.00 & 3450 \\
\hline 64 & $133 \times 12 \times 400$ & 172.2 & 336 & 1.067 & 0.00 & 3500 \\
\hline 65 & $133 \times 12 \times 400$ & 172.2 & 336 & 1.067 & 0.00 & 3650 \\
\hline 66 & $110 \times 5 \times 300$ & 133.9 & 310 & 0.562 & 0.00 & 1580 \\
\hline 67 & $113 \times 6.5 \times 300$ & 133.9 & 321 & 0.768 & 0.00 & 2076 \\
\hline 68 & $113 \times 6.5 \times 300$ & 133.9 & 321 & 0.768 & 0.00 & 2048 \\
\hline 69 & $110 \times 5 \times 300$ & 125.5 & 310 & 0.552 & 0.50 & 1620 \\
\hline 70 & $110 \times 5 \times 300$ & 125.5 & 310 & 0.552 & 0.50 & 1621 \\
\hline 71 & $110 \times 5 \times 300$ & 125.5 & 320 & 0.57 & 0.50 & 1645 \\
\hline 72 & $110 \times 5 \times 300$ & 125.5 & 320 & 0.57 & 0.50 & 1709 \\
\hline 73 & $110 \times 5 \times 300$ & 125.5 & 320 & 0.57 & 0.50 & 1655 \\
\hline 74 & $113 \times 6.5 \times 300$ & 125.5 & 321 & 0.754 & 0.50 & 2096 \\
\hline 75 & $113 \times 6.5 \times 300$ & 125.5 & 321 & 0.754 & 0.50 & 2172 \\
\hline 76 & $113 \times 6.5 \times 300$ & 163 & 321 & 0.65 & 0.00 & 2379 \\
\hline 77 & $113 \times 6.5 \times 300$ & 163 & 321 & 0.65 & 0.00 & 2415 \\
\hline 78 & $108 \times 6 \times 300$ & 170 & 391 & 0.72 & 1.00 & 2141 \\
\hline 79 & $108 \times 6 \times 300$ & 170 & 391 & 0.72 & 1.00 & 2146 \\
\hline 80 & $110 \times 5 \times 300$ & 178.2 & 320 & 0.464 & 2.00 & 1693 \\
\hline 81 & $110 \times 5 \times 300$ & 178.2 & 320 & 0.464 & 2.00 & 1641 \\
\hline 82 & $110 \times 5 \times 300$ & 178.2 & 320 & 0.464 & 2.00 & 1734 \\
\hline 83 & $110 \times 5 \times 300$ & 125.5 & 310 & 0.552 & 0.50 & 1789 \\
\hline 84 & $110 \times 5 \times 300$ & 125.5 & 310 & 0.552 & 0.50 & 1730 \\
\hline 85 & $110 \times 5 \times 300$ & 125.5 & 320 & 0.57 & 0.50 & 1865 \\
\hline 86 & $110 \times 5 \times 300$ & 125.5 & 320 & 0.57 & 0.50 & 1745 \\
\hline 87 & $110 \times 5 \times 300$ & 178.2 & 320 & 0.464 & 2.00 & 1886 \\
\hline 88 & $110 \times 5 \times 300$ & 178.2 & 320 & 0.464 & 2.00 & 1745 \\
\hline 89 & $110 \times 5 \times 300$ & 133.9 & 310 & 0.562 & 0.00 & 1789 \\
\hline 90 & $110 \times 5 \times 300$ & 125.5 & 310 & 0.552 & 0.50 & 1760 \\
\hline 91 & $110 \times 5 \times 300$ & 125.5 & 310 & 0.552 & 0.50 & 1780 \\
\hline 92 & $108 \times 6 \times 300$ & 170 & 391 & 0.72 & 1.00 & 2127 \\
\hline 93 & $108 \times 6 \times 300$ & 170 & 391 & 0.72 & 1.00 & 2170 \\
\hline 94 & $108 \times 6 \times 300$ & 170 & 391 & 0.72 & 1.00 & 2034 \\
\hline 95 & $108 \times 6 \times 300$ & 170 & 391 & 0.72 & 1.00 & 2090 \\
\hline 96 & $152 \times 5 \times 500$ & 87.4 & 314 & 0.595 & 0.00 & 2704 \\
\hline 97 & $152 \times 5 \times 500$ & 87.4 & 314 & 0.595 & 0.00 & 2679 \\
\hline 98 & $152 \times 5 \times 500$ & 87.4 & 314 & 0.595 & 0.00 & 2799 \\
\hline 99 & $152 \times 5 \times 500$ & 76.6 & 314 & 0.639 & 0.00 & 2287 \\
\hline 100 & $152 \times 5 \times 500$ & 76.6 & 314 & 0.639 & 0.00 & 2306 \\
\hline 101 & $121 \times 6 \times 450$ & 85.4 & 316 & 0.979 & 0.00 & 1907 \\
\hline 102 & $121 \times 6 \times 450$ & 85.4 & 316 & 0.979 & 0.00 & 1902 \\
\hline 103 & $102 \times 4 \times 450$ & 85.4 & 291 & 0.689 & 0.00 & 1183 \\
\hline 104 & $102 \times 4 \times 450$ & 85.4 & 291 & 0.689 & 0.00 & 1140 \\
\hline 105 & $152 \times 5 \times 450$ & 85.4 & 322 & 0.625 & 0.00 & 2833 \\
\hline 106 & $152 \times 5 \times 450$ & 85.4 & 322 & 0.625 & 0.00 & 3057 \\
\hline 107 & $121 \times 6 \times 450$ & 113.6 & 316 & 0.734 & 0.00 & 2165 \\
\hline 108 & $121 \times 6 \times 450$ & 113.6 & 316 & 0.734 & 0.00 & 2173 \\
\hline 109 & $102 \times 4 \times 450$ & 113.6 & 291 & 0.517 & 0.00 & 1326 \\
\hline 110 & $102 \times 4 \times 450$ & 113.6 & 291 & 0.517 & 0.00 & 1275 \\
\hline 111 & $152 \times 5 \times 450$ & 113.6 & 322 & 0.469 & 0.00 & 2961 \\
\hline 112 & $152 \times 5 \times 450$ & 113.6 & 322 & 0.469 & 0.00 & 2870 \\
\hline 113 & $133 \times 4.5 \times 400$ & 96.8 & 351 & 0.621 & 0.00 & 1817 \\
\hline 114 & $133 \times 4.5 \times 400$ & 96.8 & 351 & 0.621 & 0.00 & 1814 \\
\hline 115 & $133 \times 4.5 \times 400$ & 96.8 & 351 & 0.621 & 0.00 & 1837 \\
\hline 116 & $133 \times 4.5 \times 400$ & 96.8 & 351 & 0.621 & 0.00 & 1843 \\
\hline 117 & $133 \times 6 \times 400$ & 96.8 & 330 & 0.808 & 0.00 & 2039 \\
\hline 118 & $133 \times 6 \times 400$ & 96.8 & 330 & 0.808 & 0.00 & 2128 \\
\hline 119 & $133 \times 6 \times 400$ & 96.6 & 330 & 0.808 & 0.00 & 1966 \\
\hline 120 & $133 \times 6 \times 400$ & 96.6 & 330 & 0.808 & 0.00 & 1978 \\
\hline
\end{tabular}


TABle 1: Continued.

\begin{tabular}{|c|c|c|c|c|c|c|}
\hline Index & Section & $f_{\mathrm{cu}}(\mathrm{MPa})$ & $f_{\mathrm{y}}(\mathrm{MPa})$ & $\xi$ & Fiber (\%) & $N_{\mathrm{u}}(\mathrm{kN})$ \\
\hline 121 & $133 \times 8 \times 400$ & 96.6 & 338 & 1.162 & 0.00 & 2550 \\
\hline 122 & $133 \times 8 \times 400$ & 96.6 & 338 & 1.162 & 0.00 & 2552 \\
\hline 123 & $133 \times 8 \times 400$ & 96.6 & 338 & 1.162 & 0.00 & 2430 \\
\hline 124 & $133 \times 8 \times 400$ & 96.6 & 338 & 1.162 & 0.00 & 2512 \\
\hline 125 & $133 \times 10 \times 400$ & 96.8 & 313 & 1.419 & 0.00 & 2890 \\
\hline 126 & $133 \times 10 \times 400$ & 96.8 & 313 & 1.419 & 0.00 & 2894 \\
\hline 127 & $133 \times 10 \times 400$ & 96.8 & 313 & 1.419 & 0.00 & 2583 \\
\hline 128 & $133 \times 10 \times 400$ & 96.8 & 313 & 1.419 & 0.00 & 2510 \\
\hline 129 & $133 \times 12 \times 400$ & 96.8 & 402 & 2.312 & 0.00 & 3374 \\
\hline 130 & $133 \times 12 \times 400$ & 96.8 & 402 & 2.312 & 0.00 & 3396 \\
\hline 131 & $133 \times 12 \times 400$ & 96.6 & 402 & 2.312 & 0.00 & 3260 \\
\hline 132 & $133 \times 12 \times 400$ & 96.6 & 402 & 2.312 & 0.00 & 3262 \\
\hline 133 & $219 \times 8 \times 657$ & 118 & 450 & 0.737 & 2.00 & 6569 \\
\hline 134 & $219 \times 10 \times 657$ & 118 & 430 & 0.908 & 2.00 & 7095 \\
\hline 135 & $219 \times 12 \times 657$ & 118 & 375 & 0.98 & 2.00 & 7318 \\
\hline 136 & $245 \times 8 \times 735$ & 118 & 425 & 0.615 & 2.00 & 7747 \\
\hline 137 & $245 \times 12 \times 735$ & 118 & 383 & 0.877 & 2.00 & 8761 \\
\hline 138 & $273 \times 8 \times 819$ & 118 & 412 & 0.529 & 2.00 & 9211 \\
\hline 139 & $273 \times 10 \times 819$ & 118 & 380 & 0.625 & 2.00 & 9666 \\
\hline
\end{tabular}

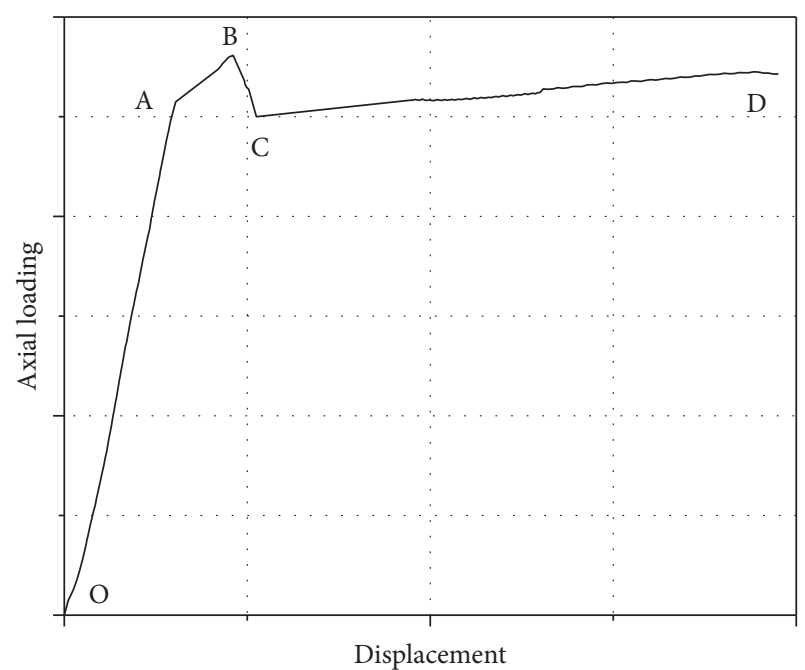

Figure 1: Load-displacement curve of the reactive powder concrete-filled steel tube (RPCFT).

bearing capacity of the steel tube was sufficient to offset the decline of the core RPC bearing capacity, with the amplitude of its rebound dependent on the confining coefficient of the specimen itself. The larger the confining coefficient, the greater the magnitude of the rebound. Yang Wusheng [10] obtained a similar conclusion when analyzing these test results, namely, under a confining coefficient $<0.4$, the bearing capacity of the specimen did not rebound and the curve was flat or continued to decrease, whereas when the bearing capacity was $>1.3$, the curve enhancement segment could experience sustainable growth.

2.2. Failure Mode. It was found that the failure modes of the 139 RPCFTs could be categorized into three types, as showed in Figure 3.

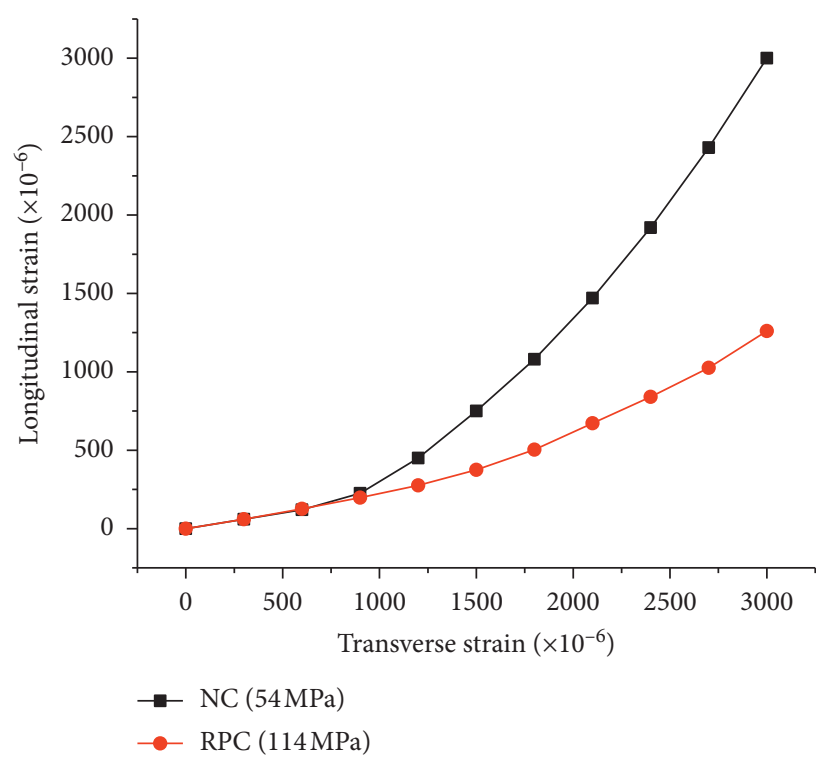

Figure 2: The transverse strain versus longitudinal strain of reactive powder concrete (RPC) and normal concrete (NC).

2.2.1. Steel Tube Buckling Failure. The CFT research results showed that under a large steel tube diameter-thickness ratio $(D / t)$, the straight steel tube underwent a "bowstring effect" under the axial pressure, and the specimen formed a "string" buckling instability by brittleness failure when the ultimate bearing capacity was reached. The CFT design specification of every country provides the $D / t$ limit, and China's "Technical Specification for CFT" (GB50936-2014) stipulates $D / t$ to be: $(20 \sim 135)\left(235 / f_{y}\right)$. However, Zhang Jing [9] studied A1-1 specimens with a diameter of $125 \mathrm{~mm}$ and a wall thickness of $1 \mathrm{~mm}$, and Yang Wusheng [10] studied specimens of a diameter of $104 \mathrm{~mm}$ and a wall thickness of $1 \mathrm{~mm}$, with $D / t$ of 125 and 104 , respectively, thereby meeting the requirements of the technical specifications for CFT. 


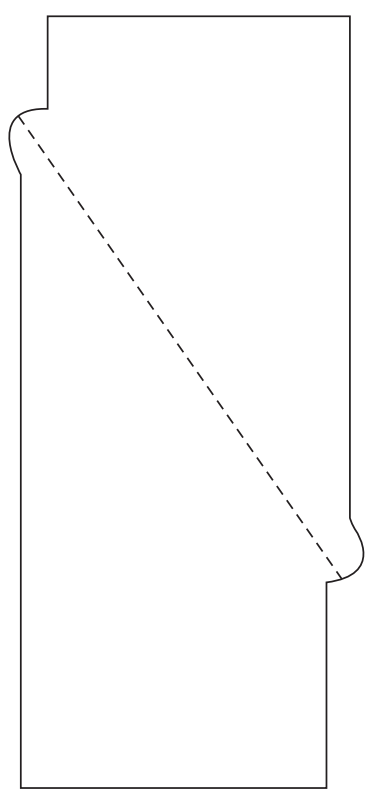

(a)

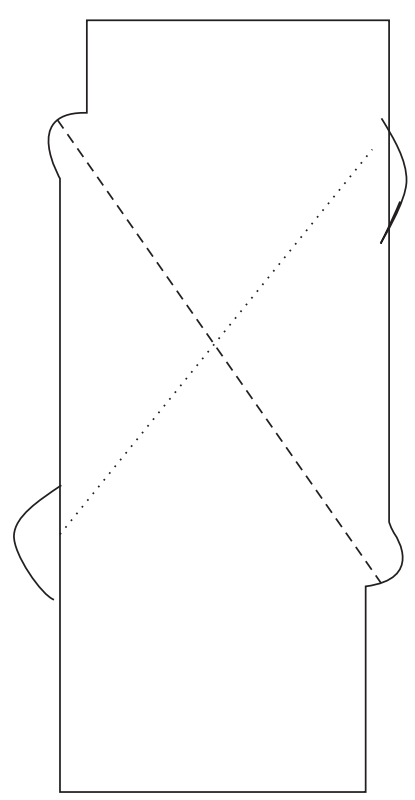

(b)
FIgURE 3: (a) Oblique shear failure and (b) waist drum-shaped failure of reactive powder concrete-filled steel tube (RPCFT).

However, "string" buckling instability remained, mainly because deformation of NC increased rapidly reaching $40 \%$ of the ultimate load, and the volume of the core concrete expanded significantly, thereby playing a certain role in supporting the steel tube wall. The transverse strain and volume expansion of RPCFT did not markedly change before reaching the ultimate load. The core concrete cannot support or restrain the tube wall. Therefore, it is recommended that the $D / t$ ratio of RPCFT be determined in accordance with the "Code for Design and Construction of CFT" (CECS 28:90) as $(20 \sim 80)\left(235 / f_{y}\right)$.

2.2.2. Oblique Shear Failure. The internal energy accumulated in the core RPC was suddenly released to form a fracture surface after the RPC reached the ultimate strength. The fracture surface generated a misalignment and formed two wedge blocks above and below, which were able to slide along the shear surface, resulting in local convexity of the steel tube. However, the increase in the confining coefficient resulted in a strengthening of the restraining effect of the steel tube, an increased restriction of sliding, a slowing down of the development speed of the internal shear crack, and a decrease in the bearing capacity of the steel tube RPC. Yang Wusheng [10] observed a significant shear slip plane after cutting the specimen with shear failure. An inclination angle of $25^{\circ}-31^{\circ}$ was evident; the steel fiber at the shear failure surface was removed, and powdery particles were observed at the RPC contact surface.

2.2.3. Drum-Shaped Failure. Under a large confining coefficient, although the upper and lower wedge blocks of core RPC were able to slide to form a shear slip plane, slide was prevented because the wedge block was placed under a large lateral constraint. The core RPC formed a second shear plane in the opposite direction, thereby increasing the transverse deformation of the specimen to form a partially roughened portion, eventually forming a multifold shear failure, i.e., drum bending in the position of the top and bottom roofs of the bearing capacity. The RPC was severely crushed and could be peeled off by hand. For NC, Zhang Sumei [17] found that CFT with a confining coefficient of approximately 0.8 would suffer from drum-type damage. The characteristics of RPC material and the results of 139 RPCFT showed that the critical confining coefficient is suggested to occur in oblique shear failure and waist drum failure.

\section{Analysis of Factors Affecting RPCFT Axial Compression Bearing Capacity}

3.1. Confining Coefficient. The confining coefficient $(\xi)$ is a comprehensive index that reflects the extent to which the core RPC is constrained by the steel tube and is the key factor affecting the ultimate bearing capacity and failure mode of the RPCFT. The fitting curve between $\xi$ and $N_{u} / A_{c} f_{c}$ by the test results of 87 RPCFT without steel fibers in Table 1 is shown in Figure 4.

Figure 4 shows that $\xi$ is proportional to $N_{u} / A_{c} f_{c}$, and the proportional factor is 1.394; however, the factors for HSC and NC were 1.8 and 2, respectively. The factor decreased with increasing strength due to the weakened confining coefficients of HSC and RPC. This was mainly because when NC was loaded to $40 \%$ of the ultimate load, expansion of microcracks in the transition zone between cement stone and coarse aggregate caused discontinuity of the displacement field, resulting in a rapid increase of the transverse deformation coefficient of the concrete. The large sum exceededs Poisson's ratio of the steel tube, and a large confining force was formed after the steel tube was extruded. The water-binder ratio was smaller, and the internal structure was denser for HSC or RPC. RPC was in particular close to an elastic material. Poisson's ratio of RPC did not change much before the RPC was loaded $80 \%-90 \%$ of the ultimate load. The transversal strain and vertical strain ratio developed proportionally. The lateral deformation coefficient of RPC struggled to exceed Poisson's ratio of the steel tube, and the confining force of the steel tube was difficult to initiate:

$$
\frac{N_{u}}{A_{c} f_{c}}=1.394 \xi+1.103 .
$$

3.2. Diameter to Thickness Ratio of Steel Tube. When applying a fixed steel tube outer diameter (D), the confining coefficient increased with increasing steel tube thickness $(\mathrm{t})$ and the ultimate bearing capacity continued to increase. Figures 5 and 6 show the fit of the relationship between $D / t$ and $N_{u} / A_{c} f_{c}$ from the results of Yang Guojing [15] and $\mathrm{Wu}$ Yanhai [11], respectively. From the figures, it is evident that, under a relatively small $D / t$, the change in $D / t$ had a greater influence on the ultimate bearing capacity, whereas under a larger $D / t$, variation of $D / t$ had little effect on the ultimate bearing capacity, and the critical $D / t$ ratio was $25-30$. 


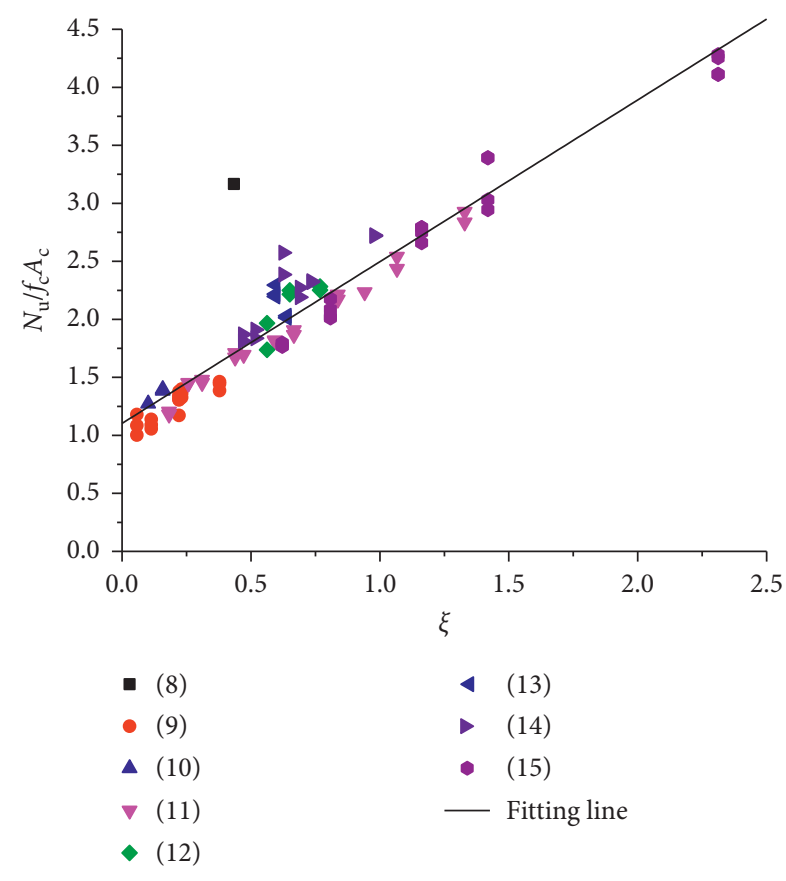

FIGURE 4: Relationship between $\xi$ and $N_{u} / A_{c} f_{c}$ by RPCFT without steel fiber.

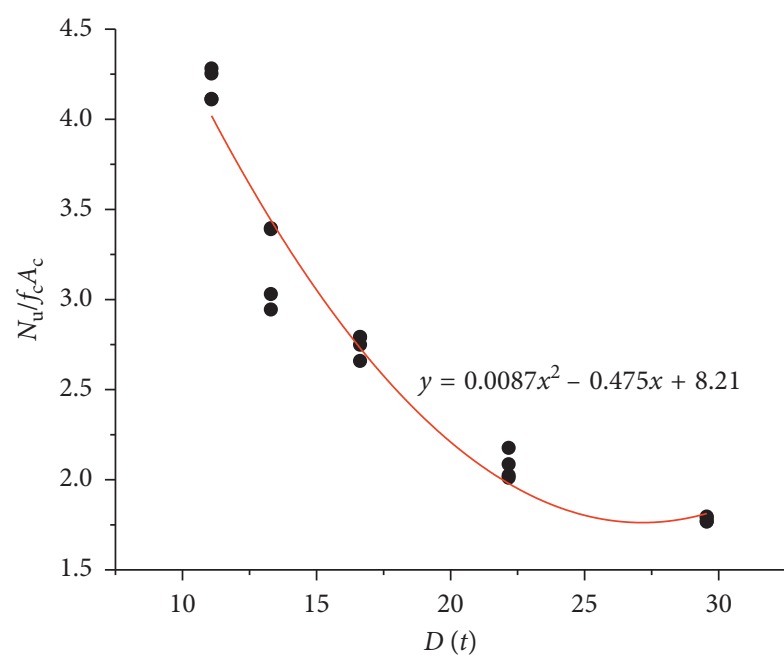

- (15)

- Fitting curve

FIGURE 5: The relationship between $D / t$ and $N_{u} / A_{c} f_{c}$ provided by Yang Guojing [15].

3.3. Core RPC Strength. Figure 7 shows the fitting relationship between $\xi$ and $N_{u} / A_{c} f_{c}$ by Yang Guojing [15] using the results of 20 RPCFT with an RPC strength of 96.6 MPa. Figure 8 shows the above relationship from the study of Zhang Jing [9] and Wu Yanhai [11] with RPC strengths of 167.1 MPa and 172.1 MPa, respectively. Evident from Figures 7 and 8 is that the scale factor between $\xi$ and $N_{u} / A_{c} f_{c}$ under a strength of $96.6 \mathrm{MPa}$ is 1.43 and under the strengths of $167.1 \mathrm{MPa}$ and $172.2 \mathrm{MPa}$ is 1.37 . Therefore, with an increase of RPC strength, the effect of the steel tube on RPC restraint was further weakened, and its proportional

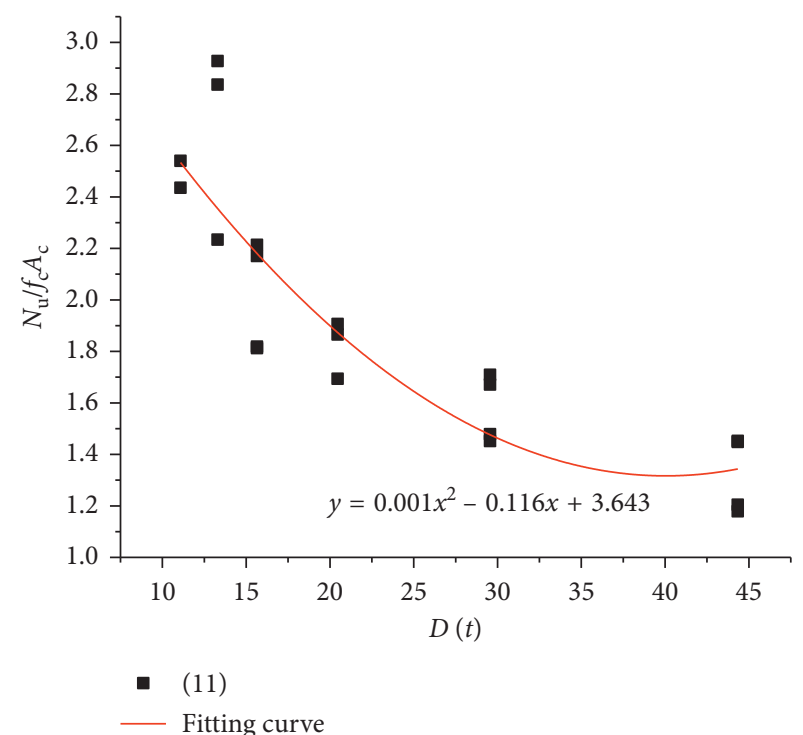

FIgURE 6: The relationship between $D / t$ and $N_{u} / A_{c} f_{c}$ provided by Wu Yanhai [11].

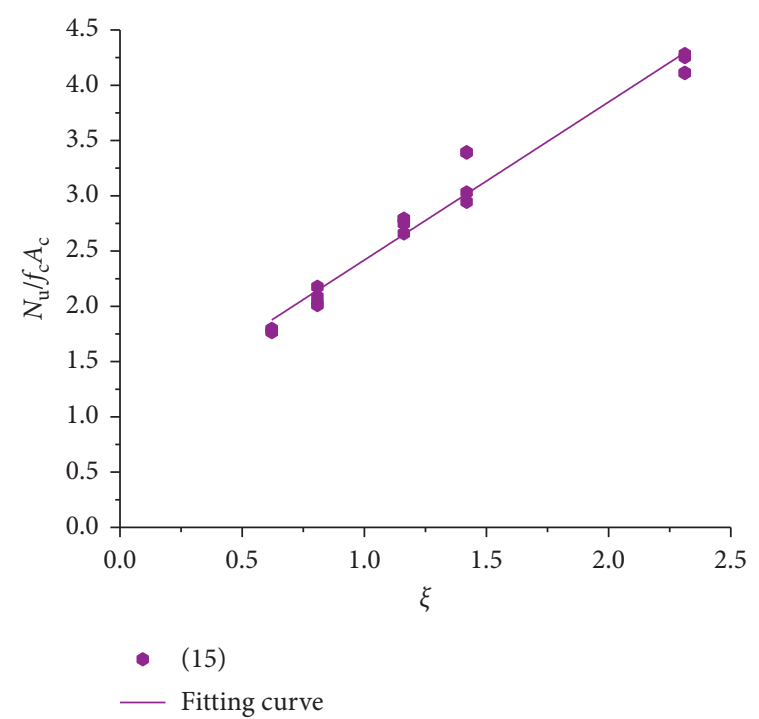

FIgURE 7: Relationship between $\xi$ and $N_{u} / A_{c} f_{c}$ provided by Yang Guojing [15].

coefficient was reduced from 1.43 to 1.37 . However, the change was merely $4.2 \%$, which can be neglected for practical applications.

3.4. Steel Fiber Content in RPC. Table 2 shows the effect of steel fiber content on the ultimate bearing capacity of RPCFT. It is evident from Table 2 that, for a steel fiber content of $1.2 \%, \mathrm{RPC}$ axial compressive strength increased by $15 \%$, and the total axial compressive strength of RPC and steel tube should be increased by approximately $10 \%$, whereas the actual ultimate load of the test samples were increased by an almost negligible amount of $0.7 \%$ to $3.8 \%$. The axial compressive strength of RPC increased by $25 \%$ under a steel fiber content of $2 \%$. Considering the contribution of RPC to strength, the theoretical ultimate capacity of 


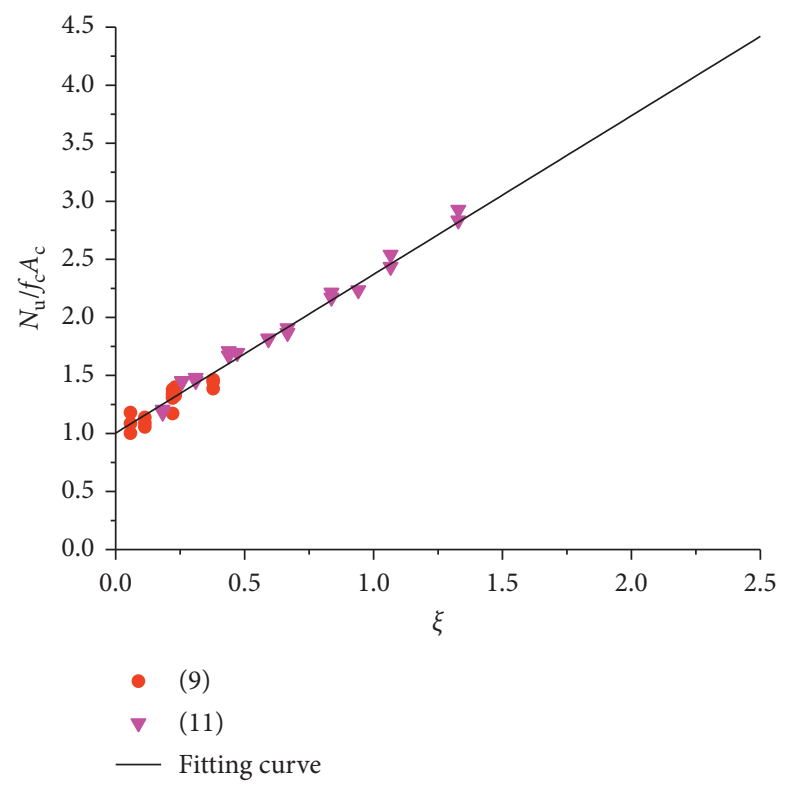

FIGURE 8: The relationship between $\xi$ and $N_{u} / A_{c} f_{c}$ provided by Zhang Jing [9].

TABLE 2: The effect of the ultimate bearing capacity by the fiber ratio in reactive powder concrete (RPC).

\begin{tabular}{|c|c|c|c|c|c|c|c|c|}
\hline Reference & Sample no. & Steel fiber (\%) & $f_{c}$ & $A_{c}$ & $N_{u}$ & $f^{\prime} / f(\%)$ & $\left(f_{c}^{\prime}-f_{c}\right) A_{c} / N_{u}(\%)$ & $N_{u}^{\prime}-N_{u} / N_{u}(\%)$ \\
\hline \multirow{2}{*}{ [8] } & B21 & 0 & 141 & 6504 & 2070 & & & \multirow{2}{*}{2.4} \\
\hline & $\mathrm{B} 22$ & 2 & - & 6504 & 2121 & & & \\
\hline \multirow{6}{*}{ [10] } & L-1 & 0 & 131 & 7854 & 1310 & \multirow{2}{*}{115} & \multirow{2}{*}{11} & \multirow{2}{*}{1.9} \\
\hline & $\mathrm{H}-1$ & 1.2 & 150 & 7854 & 1335 & & & \\
\hline & $\mathrm{L}-2 \mathrm{~A}$ & 0 & 131 & 12076 & 2190 & \multirow{2}{*}{115} & \multirow{2}{*}{10} & \multirow{2}{*}{3.0} \\
\hline & $\mathrm{H}-8 \mathrm{~A}$ & 1.2 & 150 & 12076 & 2256 & & & \\
\hline & $\mathrm{L}-2 \mathrm{~B}$ & 0 & 131 & 12076 & 2213 & \multirow{2}{*}{115} & \multirow{2}{*}{10} & \multirow{2}{*}{0.7} \\
\hline & $\mathrm{H}-8 \mathrm{~B}$ & 1.2 & 150 & 12076 & 2228 & & & \\
\hline \multirow{2}{*}{ [12] } & CFT1 & 0 & 116 & 7854 & 1580 & \multirow{2}{*}{125} & \multirow{2}{*}{14} & \multirow{2}{*}{3.8} \\
\hline & CFT8-2 & 2 & 145 & 7854 & 1641 & & & \\
\hline
\end{tabular}

RPCFT resulting from an increase of the axial compressive strength of RPC increased by approximately $14 \%$, whereas the ultimate load bearing capacity of the actual test increased by merely from $2.4 \%$ to $3.8 \%$. Almost no effect on the ultimate bearing capacity could be expected. Therefore, although the steel fiber content could increase the axial compressive strength of RPC, the contribution to the ultimate bearing capacity of RPCFT was negligible, consistent with the conclusion of Dallaier [18], mainly because of differences in the action mechanism between steel fibers and steel tubes in RPC. The main role of steel fiber was to withstand some of the tensile stress placed on the RPC principal stress section, thereby increasing the strength of RPC. However, the core RPC confined by the steel tube was compressed in three directions, and the principal compressive stress failure was found when the ultimate load reached. Therefore, the addition of steel fiber to steel tube RPC did not contribute considerably to the bearing capacity. To reduce costs, the addition of steel fiber to RPCFT is not recommended.

3.5. Loading Method. The most commonly used loading methods for CFT as shown in Figure 9 include full-section load and core-loaded concrete. Table 3 compares the ultimate bearing capacity from the study of Yang Guojing [15] under different loading methods. The differences in bearing capacities under both loading modes were within 5\%. It can be considered that the loading method was applied to the mechanical properties of the axially compressed short columns of steel tube RPC. The impact could be regarded as negligible.

\section{Analysis of RPCFT-Bearing Capacity Based on Limit Equilibrium Theory}

Methods of determining the ultimate bearing capacity of CFT can be divided into two categories: (1) numerical analysis methods and (2) limit analysis methods [19]. A numerical analysis method analyzes the material stressstrain relationship to simulate the load history and process, thereby determining the force and deformation characteristics of the entire process from elasticity to failure. The limit analysis method is based on the force balance relationship evident when components are in a limit bearing capacity state. This method can calculate the ultimate bearing capacity and is independent of the loading history and the deformation process. At present, research on the 


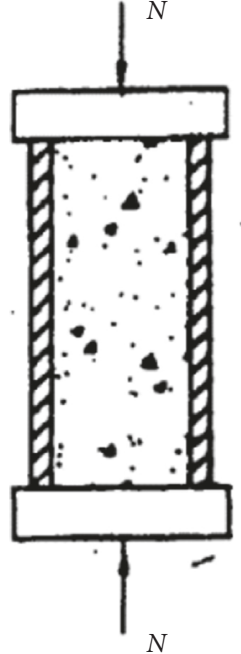

(a)

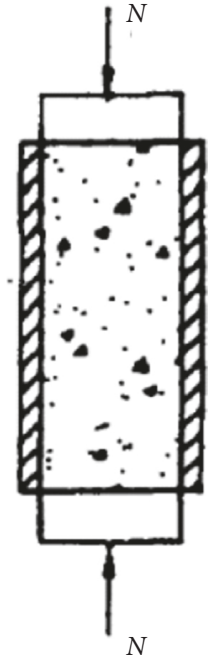

(b)
FIGURE 9: Loading pattern of the RPC-filled circular tube used in the experiment. (a) Loading on the reactive powder concrete (RPC) and tube. (b) Loading only on the reactive powder concrete (RPC).

TABLE 3: Effect of the ultimate bearing capacity according to the loading pattern.

\begin{tabular}{lcccc}
\hline Sample no. & Loading pattern & $\xi$ & $N_{u}$ & $N_{u}(A) / N_{u}(B)(\%)$ \\
\hline A-4.1 & A & 0.621 & 1817 & \\
A-4.2 & A & 0.621 & 1814 & 99 \\
B-4.1 & B & 0.621 & 1837 & \\
B-4.2 & B & 0.621 & 1843 & \\
\hline A-6-1 & A & 0.808 & 2039 & \\
A-6-2 & A & 0.808 & 2128 & 106 \\
$-6-1$ & B & 0.808 & 1966 & \\
B-6-2 & B & 0.808 & 1978 & \\
\hline A-8-1 & A & 1.162 & 2550 & \\
A-8-2 & A & 1.162 & 2552 & 103 \\
B-8-1 & B & 1.162 & 2430 & \\
B-8-2 & B & 1.162 & 2512 & \\
\hline A-12-1 & A & 2.312 & 3374 & \\
A-12-2 & A & 2.312 & 3396 & 104 \\
B-12-1 & B & 2.312 & 3260 & \\
B-12-2 & B & 2.312 & 3262 & \\
\hline
\end{tabular}

stress-strain relation of RPC remains inadequate, and no constitutive relationship is available to form a widely accepted constraint for RPC. Thus, this method does not meet the conditions for numerical analysis. Therefore, the limit analysis method was used to explore the ultimate bearing capacity of steel RPCFT axially compressed short columns.

The parameters are shown in Figure 10 so that the RPC core area and steel tube area are

$$
\begin{aligned}
& A_{c}=\frac{\pi d_{c}^{2}}{4}, \\
& A_{s}=\frac{\pi\left(D^{2}-d_{c}^{2}\right)}{4} \approx \pi d_{c} t .
\end{aligned}
$$

According to the internal force balance, the relationship between annular tension and confining force of the steel tube is

$$
\sigma_{r} d_{c}=-2 \sigma_{2} t
$$

Synthesis (equations (2)-(4)) can be obtained:

$$
\sigma_{2}=-\frac{\sigma_{r} d_{c}}{2 t}=-\sigma_{r} \frac{2 A_{c}}{A_{s}} .
$$

The steel tube conforms to the von Mises yield criterion, which is

$$
\sigma_{1}^{2}+\sigma_{2}^{2}-\sigma_{1} \sigma_{2}=f_{y}^{2}
$$

Equation (6) can be solved as follows:

$$
\sigma_{1}=\sqrt{f_{y}^{2}-\frac{3}{4} \sigma_{2}^{2}}+\frac{\sigma_{2}}{2}
$$

The study of confined concrete showed that there is a linear relationship between confined concrete strength and confining force:

$$
f_{3}=f_{c}+k \sigma_{r}
$$

Therefore,

$$
\begin{aligned}
N & =\sigma_{c} A_{c}+\sigma_{1} A_{s}, \\
& =f_{c}\left(1+k \frac{\sigma_{r}}{f_{c}}\right) A_{c}+\left(\sqrt{f_{y}^{2}-\frac{3}{4} \sigma_{2}^{2}}+\frac{\sigma_{2}}{2}\right) A_{s}, \\
& =f_{c} A_{c}+k \sigma_{r} A_{c}+\sqrt{f_{y}^{2}-\frac{3}{4}\left(-\sigma_{r} \frac{2 A_{c}}{A_{s}}\right)^{2}}-\left(-\sigma_{r} \frac{A_{c}}{A_{s}}\right) A_{s}, \\
& =\left[f_{c}+(k-1) \sigma_{r}\right] A_{c}+\sqrt{f_{y}^{2} A_{s}^{2}-3 \sigma_{r}^{2} A_{c}^{2}} .
\end{aligned}
$$

When $N$ reached the ultimate bearing capacity:

$$
\frac{d_{N}}{d_{\sigma_{r}}}=0 .
$$

That is, under equation (10), when the ultimate bearing capacity was reached, the confining force was:

$$
\sigma_{r}=\frac{f_{y} A_{s}}{A_{c}} \frac{k-1}{\sqrt{9+3(k-1)^{2}}} .
$$

Substituting confining force into equation (9):

$$
\begin{aligned}
N= & f_{c} A_{c}+f_{y} A_{s} \frac{(k-1)^{2}+3}{\sqrt{9+3(k-1)^{2}}}=f_{c} A_{c} \\
& +f_{y} A_{s} \sqrt{\frac{(k-1)^{2}+3}{3}},
\end{aligned}
$$

replaced in (12), 


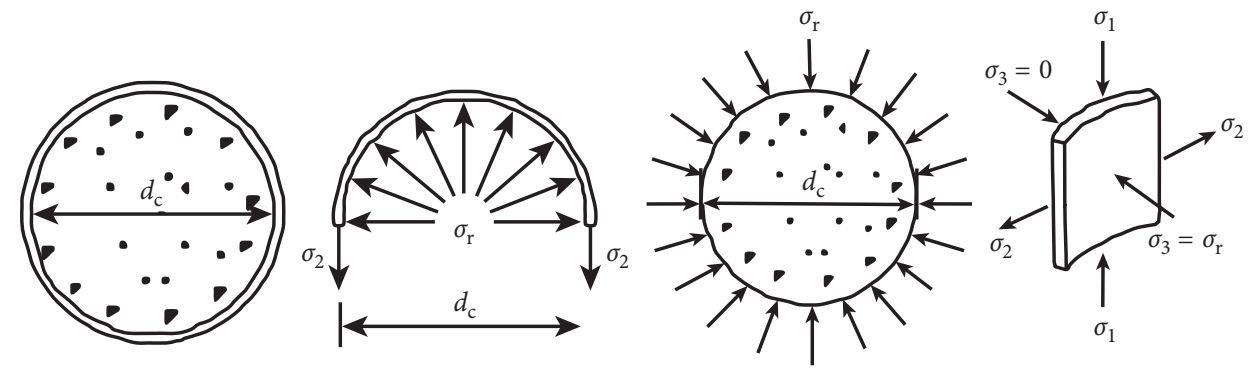

FIgURE 10: The internal stress of RPC-infilled circular tube.

$$
N_{u}=f_{c} A_{c}\left(1+\sqrt{\frac{(k-1)^{2}+3}{3}} \xi\right) .
$$

\section{Calculation of Bearing Capacity of RPCFT}

By referring to the "Technical Specifications for ConcreteFilled Steel Tubular Structures" (GB50936-2014) and the analysis results presented in Figure 4 and Equation (13), it is recommended that the value of RPC should be 1.394. The equation for calculating the bearing capacity of RPCFT is

$$
N=0.9 A_{c} f_{c}(1+1.394 \xi) \text {. }
$$

\section{Conclusion}

Circular steel tube-confined RPC is an effective method to increase ductility, and the following conclusions could be drawn from the results obtained for the analyzes:

(1) Results for a total of 139 RPCFT axial compression columns showed that the load-displacement curves can be divided into four stages: (1) elastic; (2) elasticplastic; (3) descending; and (4) strengthening. Moreover, the elastic limit could reach the ultimate limit of $90 \%$. The failure modes could be divided into three categories: (1) wall buckling instability failure; (2) oblique shear failure; and (3) waist drum damage. It was suggested that the $D / t$ ratio of RPCFT should be kept below $80\left(235 / f_{\mathrm{y}}\right)$ to avoid buckling and brittle failure of the tube wall. Oblique shear failure and drum-type damage could both result in ductile failure, and it was recommended that the critical confining coefficient be set to 1 .

(2) The larger the confining coefficient, the greater the increase in bearing capacity of RPCFT. The core RPC strength, loading method, and steel fiber content of RPC had little effect on the RPC bearing capacity of the steel tube. To reduce cost, it was recommended that no steel fiber be added to the steel tube RPC.

(3) Steel tube has less of a constraining effect on RPC than NC and HSC. The increase in the coefficient of steel tube-constrained RPC was 1.394. The equation for the ultimate bearing capacity of the steel tube
RPC was proposed in reference to the "Technical Specifications for Concrete-Filled Steel Tube Structure" (GB50936-2014).

\section{Data Availability}

The data used to support the findings of this study are available from the corresponding author upon request.

\section{Conflicts of Interest}

The authors declare that there are no conflicts of interest regarding the publication of this paper.

\section{Acknowledgments}

The author also would like to acknowledge the support provided by the National Natural Science Foundation of China (no. 51678190) for partially funding this research. All staff and laboratory technicians are thanked for their kind assistance during the research.

\section{References}

[1] P. Richard and M. Cheyrezy, "Composition of reactive powder concretes," Cement and Concrete Research, vol. 25, no. 7, pp. 1501-1511, 1995.

[2] P. Richard and M. Cheyrezy, "Reactive powder concretes with high ductility and 200-800 MPa compressive strength," ACI Spring Conversion, vol. 114, pp. 507-518, 1994.

[3] W. Zheng and L. Li, "Preparation and mix proportion calculation of reactive powder concrete," Journal of Hunan University (Science), vol. 36, no. 2, pp. 13-17, 2009.

[4] W. Zheng and LV. XueYuan, "Literature review of reactive powder concrete," Journal of Building Structures, vol. 36, no. 10, pp. 44-58, 2015.

[5] X. Hou, S. Cao, Q. Rong, W. Zheng, and G. Li, "Effects of steel fiber and strain rate on the dynamic compressive stress-strain relationship in reactive powder concrete," Construction and Building Materials, vol. 170, pp. 570-581, 2018.

[6] L. Han, Structure of Concrete Filled Steel Tube, Science Press, Beijing, China, 2004.

[7] P. Y. Blais and M. Couture, "Precast, prestressed pedestrian Bridge world's first reactive powder concrete structure," PCI Journal, vol. 44, no. 5, pp. 60-71, 1999.

[8] M. Shiqiang, "Preliminary study of reactive powder concrete filled steel tube," China Concrete and Cement Products, vol. 1, no. 1, pp. 5-8, 2003. 
[9] J. Zhang, Experimental Investigation On Behavior Of Reactive Powder Concrete Filled Steel Tube, Fuzhou University, Fuzhou, China, 2003.

[10] W. Yang, The Mechanic Property and Ultimate Bearing Capacity of Reactive Powder Concrete Filled Steel Tube, Hunan University, Changsha, China, 2003.

[11] Y. Wu and L. Yao, "Experimental study of axial compressive performance of reactive powder concrete filled steel tube," Building Structure: China, vol. 39, no. 1, pp. 821-823, 2009.

[12] J. Feng, Study on Mechanical Behavior of Reactive Powder Concrete Filled Steel Tubular Columns, Tsinghua University, Beijing, China, 2008.

[13] Z. Yan, W. Zhang, and A. Mingzhe, "The analysis of reactive powder concrete filled steel tubular columns ultimate bearing capacity," Journal of Beijing University of Technology, vol. 37, no. 3, pp. 361-367, 2011.

[14] H. Luo, Research on Behavior of Reactive Powder ConcreteFilled Circular Steel Tube Stub Columns under Aixal Compression, Beijing Jiatong University, Beijing, China, 2011.

[15] G. Yang, Research on Behavior and Ultimate Bearing Capacity of Reactive Powder Concrete-Filled Steel Tube Columns under Axial Compression, Beijing Jiatong University, Beijing, China, 2013.

[16] W. Jian, Experiment Research and analysis on Behavior of Reactive Powder Concrete-filled Circular, Steel Tube Columns under Axial Compression, Harbin Institute of Technology, Harbin, China, 2013.

[17] S. Zhang, "Failure modes of short columns of high-strength concrete-filled steel tubes," China Civil Engineering Journal, vol. 37, no. 9, pp. 1-10, 2004.

[18] H. Musha, H. Ohkuma, and T. Kitamura, Innovative UFC Structures in Japan, International Symposium on Ul-tra-High Performance Fibre-Reinforced Concrete, RILEM Publications, Mar-seille, France, 2013.

[19] S. Zhong, Structure of Concrete Filled Steel Tube, Tsinghua University Press, Beijing, China, 2003. 


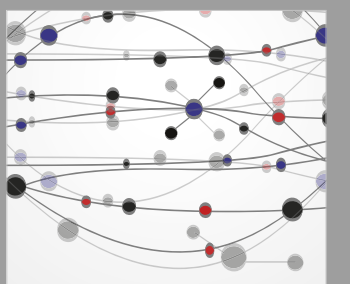

The Scientific World Journal
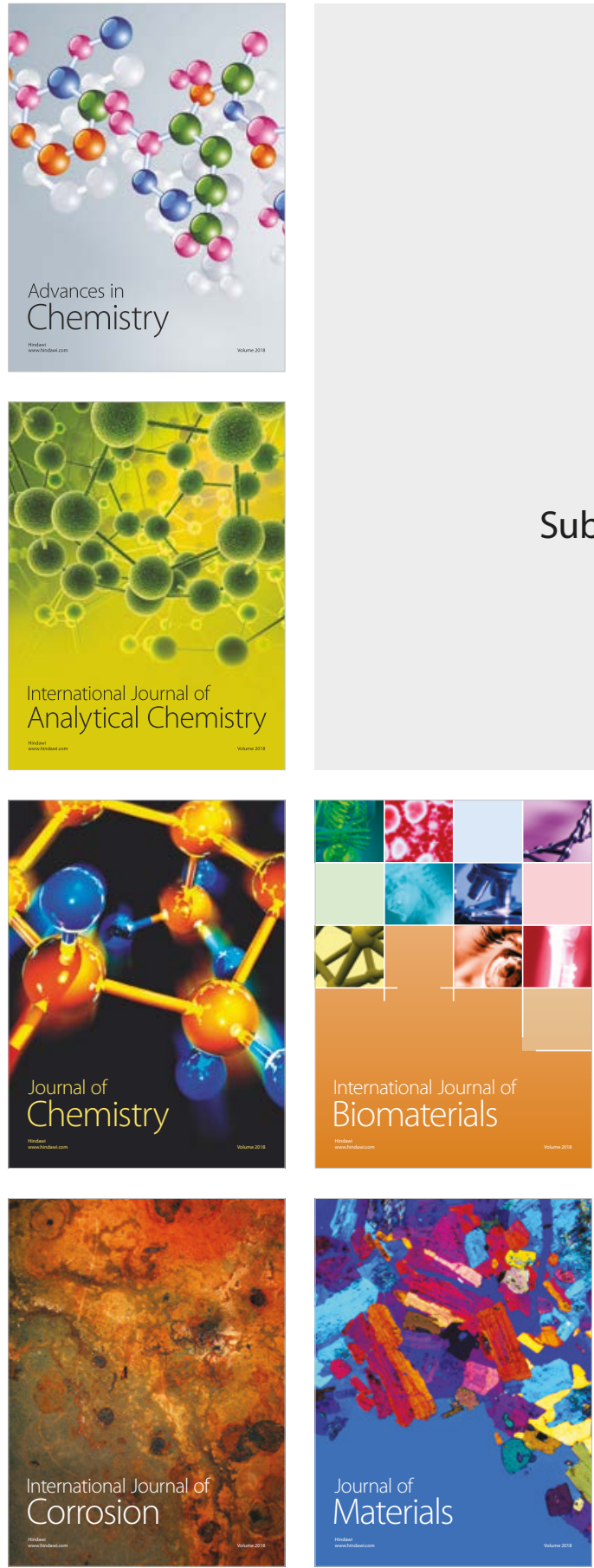

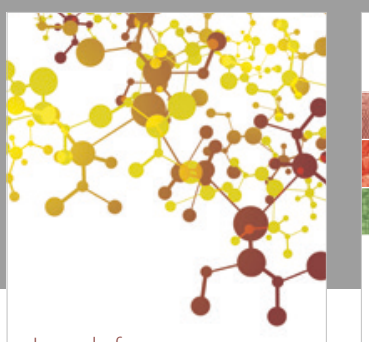

Journal of

Applied Chemistry
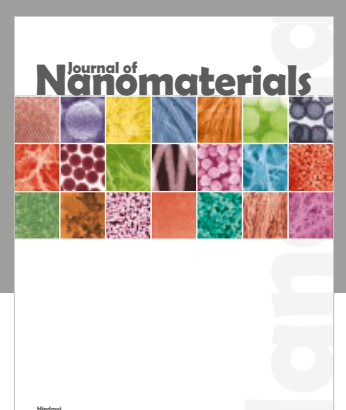

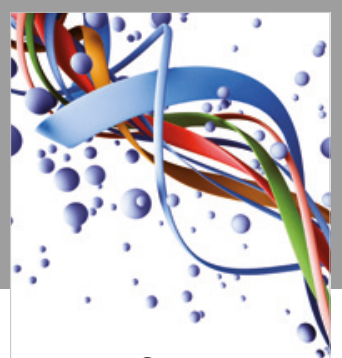

Scientifica

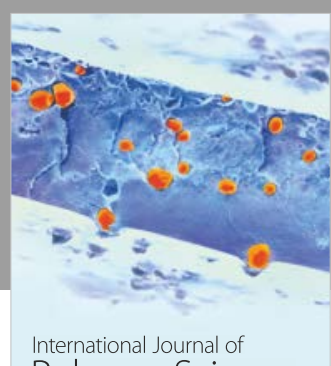

Polymer Science

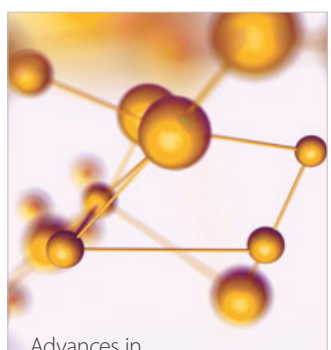

Physical Chemistry
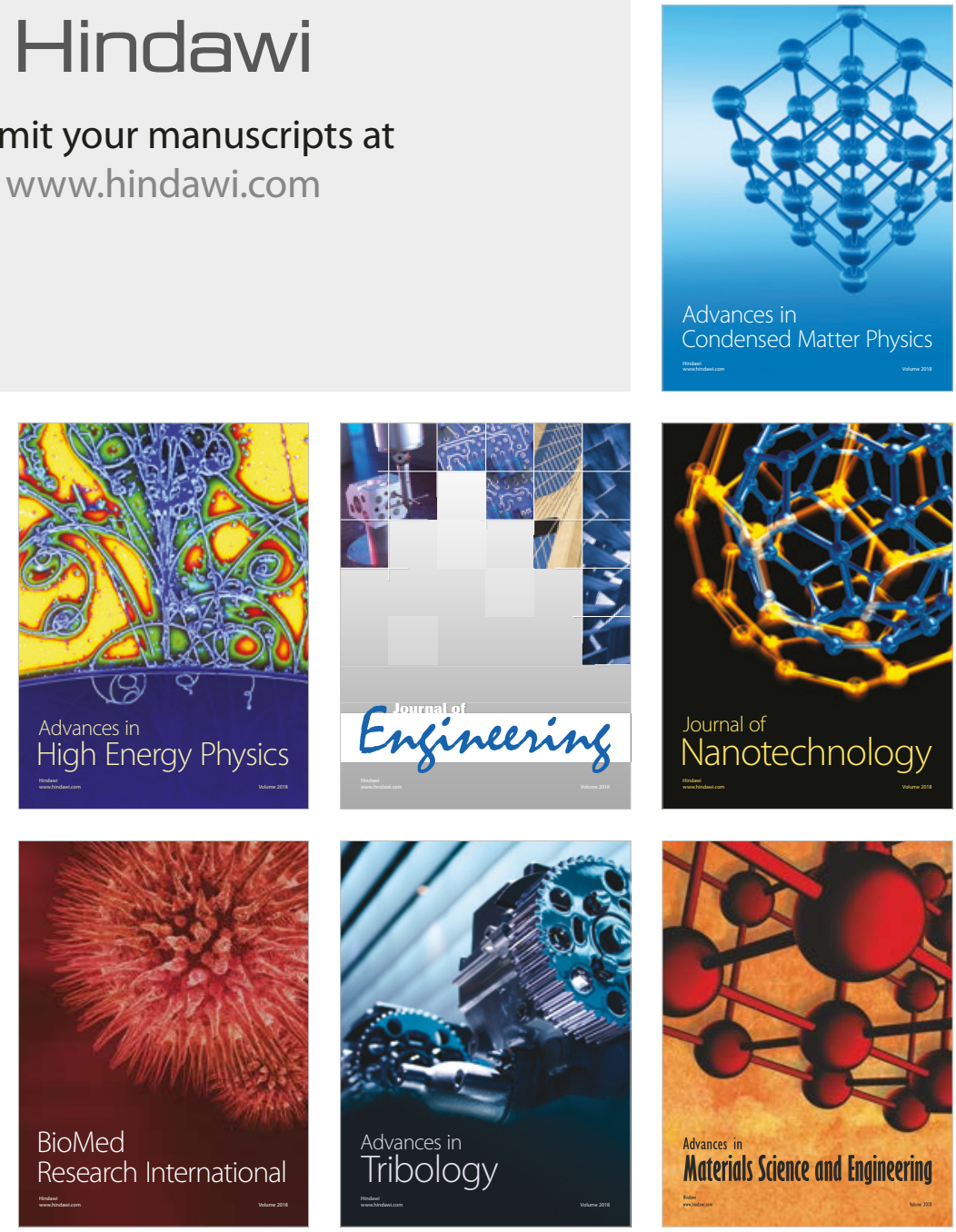